\title{
Transient Left Ventricular Apical Ballooning in a Postmenopausal Woman with Chronic Liver Disease, Malignancy, and Recent Ischemic Stroke: A Case Report and Review of the Literature
}

\author{
Dustin Covell $^{1}$ and Nathan Clendenen ${ }^{2}$ \\ ${ }^{1}$ Ross University School of Medicine, 630 US Highway 1, North Brunswick, NJ 08902, USA \\ ${ }^{2}$ Department of Medicine, St. Mary's Hospital, 56 Franklin Street, Waterbury, CT 06706, USA
}

Correspondence should be addressed to Dustin Covell, dustincovell@gmail.com

Received 29 September 2012; Accepted 29 October 2012

Academic Editors: M. Aschermann and M. R. Prince

Copyright (C) 2012 D. Covell and N. Clendenen. This is an open access article distributed under the Creative Commons Attribution License, which permits unrestricted use, distribution, and reproduction in any medium, provided the original work is properly cited.

\begin{abstract}
Transient left ventricular apical ballooning (TLVAB), or Takotsubo cardiomyopathy, is a poorly understood phenomenon that is thought to be related to a surge of catecholamines under stress conditions that causes microvascular dysfunction and a unique pattern of myocardial stunning. TLVAB occurs in two distinct patient populations: (i) community presentation similar to acute coronary syndrome and (ii) the critically ill. Understanding the differences in presentation, prognosis and management between these two groups may improve the outcome. We present a case of TLVAB developing in a Caucasian postmenopausal female who developed TLVAB suddenly on a background of chronic liver disease, malignancy, and recent ischemic stroke. The patient presented with hemodynamic collapse and was treated with inotropes, vasopressors, and supportive care until cardiac function improved. Additionally, we review the current literature describing the risk factors, pathophysiology, and treatment of TLVAB.
\end{abstract}

\section{Case Report}

A 58-year-old postmenopausal woman presented to the emergency department with a sudden onset of weakness associated with dyspnea, peripheral cyanosis, and bilateral lower extremity edema that progressed over one to two hours to include a decreased level of consciousness. In the emergency department, she was noted to be hypotensive and hypoxic. She was emergently intubated and admitted to the critical care unit. She has no significant medical history, but she reported a 25-pack-year history of smoking and drinks approximately 50 to 60 units of alcohol per week. Laboratory investigations revealed leukocytosis, thrombocytopenia, and evidence of chronic liver disease. Toxicology screen was negative for cocaine. EKG on admission was significant only for sinus tachycardia and QT prolongation. During the course of two days, the patient developed mild ST segment elevation in $\mathrm{V}_{1}$ and $\mathrm{T}$-wave inversions in leads avL, $\mathrm{I}$, and $\mathrm{V}_{1}$. No pathological $\mathrm{Q}$ waves were noted. Imaging revealed no acute intracranial hemorrhage; however, changes consistent with recent ischemic stroke were present. Additional imaging demonstrated the presence of abdominal ascites, anasarca, and a right sided pleural effusion with left upper lobe mass suspicious for malignancy. Transthoracic echocardiography demonstrated akinetic apex, hypokinetic mid-left ventricular, and hyperkinetic basal left ventricular segments (Figure 1). Left ventricular ejection fraction was $20-25 \%$ and moderate mitral regurgitation with concomitant left atrial enlargement was noted. Cardiac enzyme peak levels were measured with a troponin of 4.92 and CK-MB of 18 (17.5\% of total CPK). Biopsy of the lung masses revealed malignancy. During the acute phase of her presentation, the patient received norepinephrine and dobutamine to augment cardiac output until her TLVAB resolved.

\section{Definition and Epidemiology}

Takotsubo cardiomyopathy was originally described by Dote et al. in the early 1990s [1]. Takotsubo cardiomyopathy 


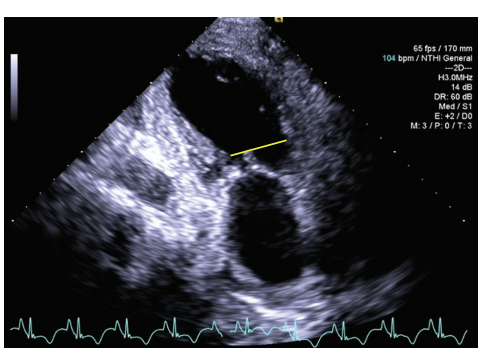

(a)

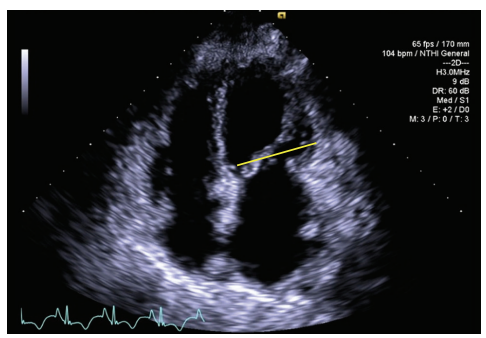

(c)

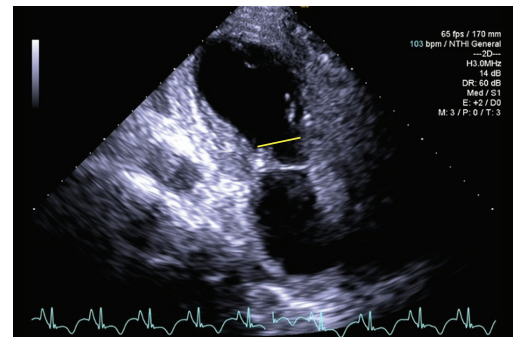

(b)

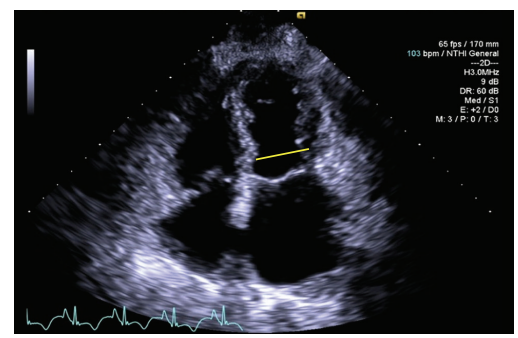

(d)

Figure 1: Two chamber transthoracic echocardiogram of the patient's LA and LV during diastole (a) and systole (b). Four chamber view during diastole (c) and systole (d). Note the absence of apical contraction during systole. Yellow lines illustrate left ventricular contraction near the mitral valve.

involves a precursor emotional or physical stress resulting in the "octopus pot" appearance of the left ventricle characterized by midventricular and apical akinesia with concomitant basal wall hyperkinesis. There are a variety of names used in the literature to describe this phenomenon: broken-heart syndrome, apical ballooning syndrome, stressinduced cardiomyopathy, in addition to other permutations of descriptors. Transient left ventricular apical ballooning (TLVAB) will be appropriated in this paper to describe this syndrome. The Mayo Criteria for TLVAB presented by Prasad et al. [2] appears to be generally accepted throughout the literature and is as follows.

(1) Transient hypokinesis, akinesis, or dyskinesis of the left ventricular mid segments with or without apical involvement; the regional wall motion abnormalities extend beyond a single epicardial vascular distribution; a stressful trigger is often, but not always present.

(2) Absence of obstructive coronary disease or angiographic evidence of acute plaque rupture.

(3) New electrocardiographic abnormalities (either STsegment elevation and/or T-wave inversion) or modest elevation in cardiac troponin.

(4) Absence of pheochromocytoma and myocarditis.

TLVAB is estimated to account for $1 \%$ of patients presenting with an acute coronary syndrome [3] and approximately 10,000 cases annually [2]. Mean patient age is $66.9 \pm$ 30.7 [4-6]. TLVAB affects women $89 \%$ of the time, and the disease has a strong predilection for Caucasians of higher socioeconomic status [5]. In critically ill patients, the incidence can be as high as $28 \%$ [7], and while women are still the dominant group, men are more likely to be affected by TLVAB in an ICU setting when compared to patients presenting with ACS [6].

When TLVAB was first studied, research was directed towards patients presenting with a predisposing emotional stressor and sudden onset of chest pain. In the last few decades, TLVAB has been discovered in association with a variety of additional physiological stressors. Anecdotal evidence supports an association between TLVAB with sepsis, malignancy, cerebrovascular accidents, asthma, pulmonary disease, respiratory distress, chronic liver disease, general anxiety disorder, alcoholism, epilepsy, subarachnoid or intracranial hemorrhage, hyperthyroidism, pheochromocytoma, recent noncardiac surgery, and dobutamine administration during stress echocardiography [8-19]. When compared to patients with myocardial infarction, TLVAB has a strong association with sepsis (OR: 3.04 [95\% CI : 2.773.334]), cerebrovascular accident (OR: 2.43 [95\% CI : $1.63-$ 3.610]), and malignancy (OR: 1.51 [CI 95\% : 1.45-1.59]) [5].

\section{Clinical Presentation and Diagnosis}

TLVAB is usually discovered in patients suspected to have an acute myocardial injury temporally related to a physiological or emotional stressor. Further investigation to rule out acute coronary syndrome is warranted when clinical suspicion is supported by positive cardiac enzymes and characteristic changes on electrocardiograph. TLVAB is often diagnosed during echocardiography or left ventriculography during angiography in the absence of significant coronary artery disease. However, among critically ill patients, presentation is subtle. Often, hemodynamic instability is attributed to 
underlying comorbidities rather than the addition of a new primary disease process.

Typical presentation of TLVAB mimics the symptoms and signs of acute coronary syndrome. Patients may report chest pain, dyspnea, and respiratory distress with or without signs of cardiogenic shock. In the critical care setting, patients may be sedated or mechanically ventilated; therefore, TLVAB manifests as acute heart failure with hemodynamic compromise. Critically ill patients are less likely to have chest pain [8], and often the first sign of TLVAB will be the presence of new onset ST segment changes, $\mathrm{T}$ wave inversions or pathological Q waves prompting inquiry of cardiac enzymes. Hemodynamic compromise develops suddenly and manifests as hypotension, tachycardia, and a decrease in stroke volume. ECG changes and cardiac enzyme elevation will be present but milder compared with STEMI patients. The unifying feature of both community and inpatient presentation of TLVAB is a temporal relationship with a physiological or emotional stressor leading to acute hemodynamic collapse.

Quantitative measures of cardiac injury in TLVAB vary in the literature. ST elevation is noted in 11-90\%, pathological Q wave in $6-31 \%$, T wave inversions (TWI) in $16-100 \%$, and QTc prolongation in $56-100 \%$ of patients $[4,7,20,21]$. Leads $V_{3}-V_{6}$ were the most common sites for ST elevation or TWI and the estimated EKG sensitivity for ST segment and $\mathrm{T}$ wave changes is $19 \%[7,8,22]$. Studies have shown that cardiac enzymes are elevated in only $46-100 \%$ of patients and the elevation is modest in comparison with true STEMI patients $[7,8]$. This wide range reflects variation between ACS patient population and ICU patient population. Left ventricular ejection fraction ranges $15-49 \%[8,23]$ with a mean LVEF of $20 \%$ [21]. Hemodynamic compromise is associated with higher peak troponin levels and lower ejection fraction on admission [23]. While there is variability in EKG findings, cardiac enzymes, and ejection fraction, most patients presenting with TLVAB will undergo cardiac workup for ACS. Clinicians should be suspicious for TLVAB in patients with aforementioned predisposing comorbidities and minimal cardiac risk factors. Compared with the patient population experiencing a myocardial infarction, patients with TLVAB are less likely to have hypertension, diabetes, past history of CAD, obesity, or a history of smoking [5].

\section{Complications}

A variety of complications may occur in the setting of TLVAB: heart failure $(31.1 \%)$, respiratory insufficiency related to pulmonary edema $(6.7 \%)$, cardiogenic shock $(4.7 \%)$, and ventricular fibrillation/cardiac arrest $(2.8 \%)$ [6]. Physicians have also documented cases of apical mural thrombus embolization [24] and septal rupture [25] in the literature. Cardiogenic shock can arise from not only TLVAB itself but also from a dynamic left ventricular outflow tract (LVOT) obstruction. Hyperkinetic basal segments and anterior systolic motion of the mitral valve can impede left ventricular outflow. Mitral valve regurgitation is often present in this dynamic obstruction. This may result in rapid deterioration of hemodynamic stability and will alter treatment strategy. LVOT obstruction has been associated with dobutamine [23] and dopamine [26] infusion; however, prompt cessation led to resolution of obstruction.

\section{Disease Mechanism}

The mechanism by which all of these predisposing factors contribute to TLVAB is not well understood. There is strong support for a causative role of catecholamines although the mechanism remains unclear. Proposed aetiologies include: multiple epicardial vessel spasm, direct myocardial damage resulting from catecholamine toxicity, neurohumoral stunning, and microvascular spasm.

Catecholamines are thought to play a crucial role in the development of TLVAB as the disease is associated with increased levels of norepinephrine, epinephrine, and dopamine when compared to gender matched patients with Killip Class 3 myocardial infarctions [21]. Upon admission, epinephrine concentrations were three to fourfold higher, norepinephrine concentrations were twofold higher, and dopamine concentrations were twofold higher than levels in gender matched patients with Killip Class 3 myocardial infarctions. Brain natriuretic peptide, neuropeptide $\mathrm{Y}$, and extraneuronal catecholamine metabolites were also found to be increased. Wittstein et al. termed the cardiac impairment in TLVAB "neurohumoral stunning" as subarachnoid hemorrhage and pheochromocytoma can also produce the syndrome. This stunning could be related to increased intracellular cAMP or calcium overload at the cardiac apex, where the myocardium is more sensitive to sympathetic stimulation compared to the rest of the heart [21]. Takashi et al. demonstrated that TLVAB could be recreated in a murine model by inducing emotional stress through immobilization. Prophylactic adrenergic blockade during immobilization prevented the development of TLVAB [27]. Although these results may not be directly extrapolated for application in patients, further study is necessary to clarify the mechanism of catecholamine involvement in TLVAB.

Direct myocardial damage from catecholamine excess is known to occur in pheochromocytoma and subarachnoid hemorrhage; however, it is not known how the catecholamine surge contributes to the formation of TLVAB. Histological appearance of tissue affected by catecholamine toxicity reveals interstitial mononuclear infiltration with contraction band necrosis. Similarly, biopsy in TLVAB has demonstrated mononuclear infiltration but contraction band myocytic necrosis is not concomitantly present $[21,22]$. In addition to direct myocardial damage from catecholamines, circulating cytokines may also influence myocardial contractility. The proinflammatory cytokines TNF $\alpha$ and IL-1 $\beta$ exert a direct depressant effect on the myocardium resulting in segmental or global hypokinesia that is distinct from the effects of isolated hypoperfusion in septic shock $[28,29]$. Proinflammatory cytokines are elevated in many other disease processes, including malignancy, and it is possible that TNF $\alpha$ and IL-1 $\beta$ contribute 
to the decrease in cardiac function noted in other disease processes [30].

Multiple epicardial coronary vessel spasm is another potential explanation for the mechanism of TLVAB; however, the data are less convincing. The rationale for this theory extends from knowledge of coronary vessel anatomy. The apex is the most distal territory supplied in a watershed area of blood supply between the main coronary arteries and is therefore more susceptible to interruption in blood flow. Vasospasm may be able to produce the unusual pattern of apical hypokinesis with basal hyperkinesis in TLVAB, although multivessel vasospasm would be required to cause myocardial stunning in the apex. While possible, this explanation is less plausible than alternative theories. Additionally, direct experimental evidence of the role of postvasospasm metabolic derangements in maintaining myocardial stunning is unclear. Results of vasospasm provocation using ergonovine or acetylcholine are variable with ergonovine or acetylcholine infusion inducing vasospasm in $21-70 \%$ of the patients $[8,22]$. Successful provocation of vasospasm has been shown to worsen TLVAB in some cases [3133]; therefore, if patients with TLVAB demonstrate reactive vasospasm, strategic management with adjunct calcium channel blocker therapy may be beneficial.

Cardiac magnetic resonance imaging (CMRI) has also been used to describe TLVAB and differentiate it from other similarly presenting illnesses. In CMRI, presence of delayed gadolinium enhancement would imply myocardial tissue necrosis which is present in myocarditis or infarction. With TLVAB, the characteristic absence of late gadolinium enhancement (LGE) demonstrates that the myocardium is viable and well perfused $[21,23]$. Vasospasm in multiple epicardial vessels would produce ischemia, thus vasospasm as the primary mechanism in TLVAB is unlikely. However, absent LGE provides little information about microvascular status.

Microvascular compromise is considered to be the most likely theory leading to TLVAB formation. Microvascular function can be assessed with positron emission tomography (PET), scintigraphy, TIMI frame counts, and myocardial blush grade; however, the evidence from these methods remains inferential. PET and CT comparison can demonstrate both perfusion and metabolic defects primarily at the apex. This led to descriptors of TLVAB as an "inverse metabolic/perfusion mismatch" $[3,34]$. The use of metaiodobenzylguanidine scintigraphy has additionally demonstrated apical defects [35]. This suggests that while perfusion is grossly intact and there is no evidence of necrosis, there is a metabolic derangement present. It is hypothesized that this defect is caused by impaired microcirculation. Coronary flow reserve reflects the ability of the coronary arteries to maximally decrease resistance; however, the most dynamic constituents are not the large epicardial vessels, but rather the arterioles penetrating the myocardium (microvasculature). Quantitative measurements of myocardial blood flow (MBF) provide direct information about coronary flow reserve [22]. In TLVAB, coronary flow reserve was found to be diffusely abnormal $[34,35]$. TIMI flow grade and myocardial blush can provide gross assessment of microcirculation and show impairment [3].

Microvascular dysfunction has been studied extensively in the context of coronary syndrome $\mathrm{X}$ where despite lack of angiographic evidence of coronary artery disease, patients have chest pain linked to microvascular damage. The use of NMR spectroscopy investigation for the release of high energy phosphate during hand grip exercises has been a topic of study for coronary syndrome $\mathrm{X}$ which may elucidate whether the microvascular impedance occurring in TLVAB is related to microvascular spasm or damage [36].

\section{Treatment}

The goal of treatment in TLVAB is to support cardiac output during the acute phase to allow the apical myocardium to recover. With the catecholamine theories in mind, beta blockers are thought to be beneficial in the treatment of TLVAB and their use has been reported across the literature. However, pulmonary edema is commonly reported in the acute phase and may later prove to be a contraindication for the use of beta blockers acutely. Aggressive diuresis is necessary if the patient's respiratory status is compromised by pulmonary edema. In the presence of hemodynamic instability, the judicious use of vasopressors is indicated; however, exogenous catecholamines should be kept at the minimum rate required to balance hypotension [21]. Use of arginine vasopressin and hydrocortisone for its permissive action on adrenergic receptors has been reported in the treatment of TLVAB [37]. If left ventricular outflow tract (LVOT) obstruction occurs, cautious fluid resuscitation and use of phenylephrine to increase afterload and chamber size has been proposed [2]. Use of catecholamine inotropes will worsen LVOT obstruction. Intra-aortic balloon pump counterpulsation is used to decrease afterload and increase myocardial perfusion and has been a mainstay of successful management in unstable patients. Levosimendan, a calcium channel sensitizer, is being reported with increasing popularity for the treatment of cardiogenic shock. It is especially useful in TLVAB as its mechanism of action does not involve catecholamine receptors. Some studies have suggested that levosimendan replaces the IABP which has been a staple in bridge therapy through the acute phase of TLVAB [37-40].

Levosimendan has a novel mechanism of action. It causes coronary vasodilation and increased contractility without causing a significant increase in oxygen consumption, intracellular cAMP or $\mathrm{Ca}^{2+}$. Levosimendan acts on ATP-sensitive $\mathrm{K}^{+}$channels, reinforces $\mathrm{Ca}^{2+}$ binding with Troponin C during systole, and improves ventricular relaxation during diastole [41]. In patients with cardiogenic shock it is found to increase Cardiac Index (CI) and Cardiac Power Index (CPI) while pulmonary artery pressure and mean arterial pressure have no significant changes [42]. Levosimendan is known to cause a reduction in blood pressure $>5 \mathrm{mmHg}$; however, preload augmentation with IV fluid bolus can be given prophylactically. The ability of levosimendan to increase cardiac index without use of adrenergic receptors is uniquely compatible with the treatment of TLVAB. 


\section{Prognosis}

Presence of underlying critical illness is a strong determinant in predicting mortality. For patients presenting with acute coronary syndrome following an emotional stressor, inhospital mortality is $1.1 \%$. However when you also consider the patient population that is acutely and critically ill, inhospital mortality is $4.2 \%[3,4,6,8]$. Among only the critically ill, in-hospital mortality increases to $12.1 \%$ and the prevalence of underlying critical illness among TLVAB patients was $27.9 \%$. In other words, the critically ill constitute approximately $30 \%$ of the total population of TLVAB patients and account for $80 \%$ of its in-hospital mortalities [6].

Males have a higher mortality rate than females $(8.4 \%$ versus $3.6 \%, P<0.0001$ ), which likely reflects the higher incidence of critical illness in males (36.6\% versus $26.8 \%$, $P<0.0001)$. Male gender is independently associated with increased mortality with an odds ratio of 2.07 and exceeds mortality among TLVAB patients with comorbid malignancy $(8.1 \%)$. Sepsis is associated with a $21.6 \%$ mortality rate in patients with TLVAB [6]. Another study found that sepsis is the only variable to have a significant independent association with the development of TLVAB [7].

If the patient is stabilized during the acute phase of the disease, wall motion abnormalities typically resolve over days to weeks [2], and ejection fraction normalizes within the first week and can double in days [21]. Complete recovery is expected within 4-8 weeks with recurrence estimated at less than $10 \%[2,3]$.

\section{Conclusion}

If we examine the patient population with critical illness as opposed to patients presenting with ACS-like syndromes, we begin to understand the impact that transient left ventricular apical ballooning can have on the course of a patient whose status is already precarious. During acute hemodynamic instability, patients with TLVAB are managed as acute heart failure patients although catecholaminergic inotropes and vasopressors have a relative contraindication. The use of levosimendan, with its novel mechanism, holds great promise for the treatment of TLVAB.

Current treatment modalities for TLVAB have not been supported by any large trials specific to TLVAB. The management is, for lack of a better word, borrowed. Further research into treatment and management of TLVAB will help decrease mortality in critically ill patient populations and may shed light on the mechanism of this unique disease process. As pooled literature is improving our understanding of the patient populations this disease occurs in, clinicians will be able to identify patients at risk and collaboratively develop a treatment protocol unique to this condition.

\section{References}

[1] K. Dote, H. Sato, H. Tateishi, T. Uchida, and M. Ishihara, "Myocardial stunning due to simultaneous multivessel coronary spasms: a review of 5 cases," Journal of Cardiology, vol. 21, no. 2, pp. 203-214, 1991.
[2] A. Prasad, A. Lerman, and C. S. Rihal, "Apical ballooning syndrome (Tako-Tsubo or stress cardiomyopathy): a mimic of acute myocardial infarction," American Heart Journal, vol. 155, no. 3, pp. 408-417, 2008.

[3] V. Kurowski, A. Kaiser, K. von Hof et al., "Apical and midventricular transient left ventricular dysfunction syndrome (tako-tsubo cardiomyopathy): frequency, mechanisms, and prognosis," Chest, vol. 132, no. 3, pp. 809-816, 2007.

[4] K. A. Bybee, T. Kara, A. Prasad et al., "Systematic review: transient left ventricular apical ballooning: a syndrome that mimics ST-segment elevation myocardial infarction," Annals of Internal Medicine, vol. 141, no. 11, pp. 858-865, 2004.

[5] A. M. El-Sayed, W. Brinjikji, and S. Salka, "Demographic and Co-morbid predictors of stress (Takotsubo) cardiomyopathy," The American Journal of Cardiology, vol. 110, no. 9, pp. 1368$1372,2012$.

[6] W. Brinjikji, A. M. El-Sayed, and S. Salka, "In-hospital mortality among patients with takotsubo cardiomyopathy: a study of the National Inpatient Sample 2008 to 2009," American Heart Journal, vol. 164, pp. 215-221.

[7] J.-H. Park, S. J. Kang, J. K. Song et al., "Left ventricular apical ballooning due to severe physical stress in patients admitted to the medical ICU," Chest, vol. 128, no. 1, pp. 296-302, 2005.

[8] K. Tsuchihashi, K. Ueshima, T. Uchida et al., "Transient left ventricular apical ballooning without coronary artery stenosis: a novel heart syndrome mimicking acute myocardial infarction," Journal of the American College of Cardiology, vol. 38 , no. 1, pp. 11-18, 2001.

[9] R. Hachamovitch, J. D. Chang, R. E. Kuntz, P. Papageorgiou, M. S. Levin, and A. L. Goldberger, "Recurrent reversible cardiogenic shock triggered by emotional distress with no obstructive coronary disease," American Heart Journal, vol. 129, no. 5, pp. 1026-1028, 1995.

[10] F. M. Sarullo, L. Americo, S. Accardo et al., "Tako-tsubo cardiomyopathy observed in a patient with sepsis and transient hyperthyroidism," Monaldi Archives for Chest Disease, vol. 72, no. 1, pp. 33-36, 2009.

[11] B. K. Kantharia, H. B. Richards, and J. Battaglia, "Reversible dilated cardiomyopathy: an unusual case of thyrotoxicosis," American Heart Journal, vol. 129, no. 5, pp. 1030-1032, 1995.

[12] S. Geng, D. Mullany, and J. F. Fraser, "Takotsubo cardiomyopathy associated with sepsis due to Streptococcus pneumoniae pneumonia," Critical Care and Resuscitation, vol. 10, no. 3, pp. 231-234, 2008.

[13] S. Ohigashi-Suzuki, Y. Saito, and I. Tatsuno, "Takotsubo cardiomyopathy associated with sepsis in type 2 diabetes mellitus," American Journal of Emergency Medicine, vol. 25, no. 2, pp. 230-232, 2007.

[14] J. R. Bennett, D. Mccarty, C. M. Wilson, and F. O’Neill, "Takotsubo cardiomyopathy: an uncommon cause of ST segment elevation in Intensive Care," Anaesthesia, vol. 62, no. 3, pp. 279-281, 2007.

[15] T. Kono, H. Morita, T. Kuroiwa, H. Onaka, H. Takatsuka, and A. Fujiwara, "Left ventricular wall motion abnormalities in patients with subarachnoid hemorrhage: neurogenic stunned myocardium," Journal of the American College of Cardiology, vol. 24, no. 3, pp. 636-640, 1994.

[16] S. H. Sardesai, A. J. Mourant, Y. Sivathandon, R. Farrow, and D. O. Gibbons, "Phaechromocytoma and catecholamine induced cardiomyopathy presenting as heart failure," British Heart Journal, vol. 63, no. 4, pp. 234-237, 1990.

[17] R. Margey, P. Diamond, H. McCann, and D. Sugrue, "Dobutamine stress echo-induced apical ballooning (Takotsubo) 
syndrome," European Journal of Echocardiography, vol. 10, no. 3, pp. 395-399, 2009.

[18] J. Cherian, S. Kothari, D. Angelis, A. Atef, B. Downey, and J. Kirkpatrick, "Atypical takotsubo cardiomyopathy: dobutamine-precipitated apical ballooning with left ventricular outflow tract obstruction," Texas Heart Institute Journal, vol. 35, no. 1, pp. 73-75, 2008.

[19] J. Guerrero, A. Majid, and A. Ernst, "Cardiogenic shock secondary to Tako-tsubo syndrome after debridement of malignant endobronchial obstruction," Chest, vol. 135, no. 1, pp. 217-220, 2009.

[20] D. Haghi, S. Fluechter, T. Suselbeck et al., "Takotsubo cardiomyopathy (acute left ventricular apical ballooning syndrome) occurring in the intensive care unit," Intensive Care Medicine, vol. 32, no. 7, pp. 1069-1074, 2006.

[21] I. S. Wittstein, D. R. Thiemann, J. A. C. Lima et al., "Neurohumoral features of myocardial stunning due to sudden emotional stress," The New England Journal of Medicine, vol. 352, no. 6, pp. 539-548, 2005.

[22] S. Kurisu, H. Sato, T. Kawagoe et al., "Tako-tsubo - like left ventricular dysfunction with ST-segment elevation: a novel cardiac syndrome mimicking acute myocardial infarction," American Heart Journal, vol. 143, no. 3, pp. 448-455, 2002.

[23] S. W. Sharkey, J. R. Lesser, A. G. Zenovich et al., "Acute and reversible cardiomyopathy provoked by stress in women from the United States," Circulation, vol. 111, no. 4, pp. 472-479, 2005.

[24] K. Yonekawa, P. Mussio, and B. Yuen, "Ischemic cerebrovascular stroke as complication of sepsis-induced Takotsubo cardiomyopathy," Internal and Emergency Medicine, vol. 6, no. 5, pp. 477-478, 2010.

[25] G. Mariscalco, P. Cattaneo, A. Rossi et al., "Tako-tsubo cardiomyopathy complicated by ventricular septal perforation and septal dissection," Heart and Vessels, vol. 25, no. 1, pp. 7375, 2010.

[26] Y. Abe, A. Tamura, and J. Kadota, "Prolonged cardiogenic shock caused by a high-dose intravenous administration of dopamine in a patient with takotsubo cardiomyopathy," International Journal of Cardiology, vol. 141, no. 1, pp. e1-e3, 2010.

[27] U. Takashi, K. Ken, H. Takuzo, Y. Katsuhiro, T. Yoshihiro, and N. Ichiro, "Emotional stress induces transient left ventricular hypocontraction in the rat via activation of cardiac adrenoceptors: a possible animal model of "tako-tsubo" cardiomyopathy," Circulation Journal, vol. 66, no. 7, pp. 712$713,2002$.

[28] O. Court, A. Kumar, J. E. Parrillo, and A. Kumar, "Clinical review: myocardial depression in sepsis and septic shock," Critical Care, vol. 6, no. 6, pp. 500-508, 2002.

[29] A. G. Ellrodt, M. S. Riedinger, and A. Kimchi, "Left ventricular performance in septic shock: reversible segmental and global abnormalities," American Heart Journal, vol. 110, no. 2, pp. 402-409, 1985.

[30] A. M. Lewis, S. Varghese, H. Xu, and H. R. Alexander, "Interleukin-1 and cancer progression: the emerging role of interleukin-1 receptor antagonist as a novel therapeutic agent in cancer treatment," Journal of Translational Medicine, vol. 4, article 48, 2006.

[31] P. Angelini, “Transient left ventricular apical ballooning: a unifying pathophysiologic theory at the edge of prinzmetal angina," Catheterization and Cardiovascular Interventions, vol. 71, no. 3, pp. 342-352, 2008.
[32] P. Angelini, "Midventricular variant of transient apical ballooning: a likely demonstration of its pathophysiologic mechanism," Mayo Clinic Proceedings, vol. 84, no. 1, pp. 92-93, 2009.

[33] P. Angelini, "Takotsubo cardiomyopathy: what is behind the octopus trap?" Texas Heart Institute Journal, vol. 37, no. 1, pp. 85-87, 2010.

[34] M. Feola, G. L. Rosso, F. Casasso et al., "Reversible inverse mismatch in transient left ventricular apical ballooning: perfusion/metabolism positron emission tomography imaging," Journal of Nuclear Cardiology, vol. 13, no. 4, pp. 587-590, 2006.

[35] K. Sadamatsu, H. Tashiro, N. Maehira, and K. Yamamoto, "Coronary microvascular abnormality in the reversible systolic dysfunction observed after noncardiac disease," Japanese Circulation Journal, vol. 64, no. 10, pp. 789-792, 2000.

[36] S. D. Buchthal, J. A. Den Hollander, C. N. B. Merz et al., "Abnormal myocardial phosphorus-31 nuclear magnetic resonance spectroscopy in women with chest pain but normal coronary angiograms," The New England Journal of Medicine, vol. 342, no. 12, pp. 829-835, 2000.

[37] C. A. Schmittinger, B. Wurzinger, M. Deutinger et al., "How to protect the heart in septic shock: a hypothesis on the pathophysiology and treatment of septic heart failure," Medical Hypotheses, vol. 74, no. 3, pp. 460-465, 2010.

[38] V. De Santis, D. Vitale, L. Tritapepe, C. Greco, and P. Pietropaoli, "Use of levosimendan for cardiogenic shock in a patient with the apical ballooning syndrome," Annals of Internal Medicine, vol. 149, no. 5, pp. 365-367, 2008.

[39] M. Karvouniaris, J. Papanikolaou, D. Makris, and E. Zakynthinos, "Sepsis-associated takotsubo cardiomyopathy can be reversed with levosimendan," The American Journal of Emergency Medicine, vol. 30, pp. 832.e5-832.e7, 2012.

[40] L. Padayachee, "Levosimendan: the inotrope of choice in cardiogenic shock secondary to takotsubo cardiomyopathy?" Heart Lung and Circulation, vol. 16, supplement 3, pp. S65S70, 2007.

[41] M. Antonini, G. V. Stazi, M. T. Cirasa, G. Garotto, and A. Frustaci, "Efficacy of levosimendan in Takotsubo-related cardiogenic shock," Acta Anaesthesiologica Scandinavica, vol. 54, no. 1, pp. 119-120, 2010.

[42] M. A. Russ, R. Prondzinsky, A. Christoph et al., "Hemodynamic improvement following levosimendan treatment in patients with acute myocardial infarction and cardiogenic shock," Critical Care Medicine, vol. 35, no. 12, pp. 2732-2739, 2007. 


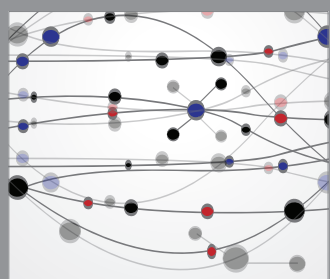

The Scientific World Journal
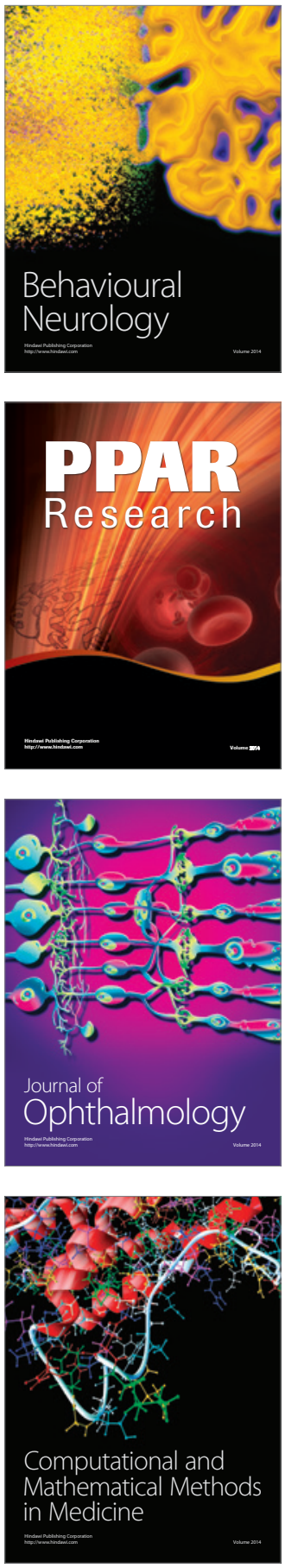

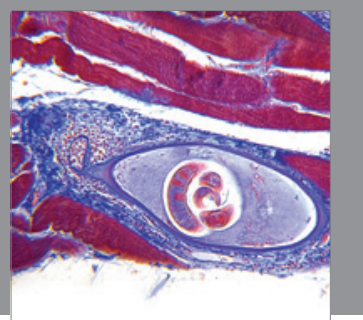

Gastroenterology

Research and Practice
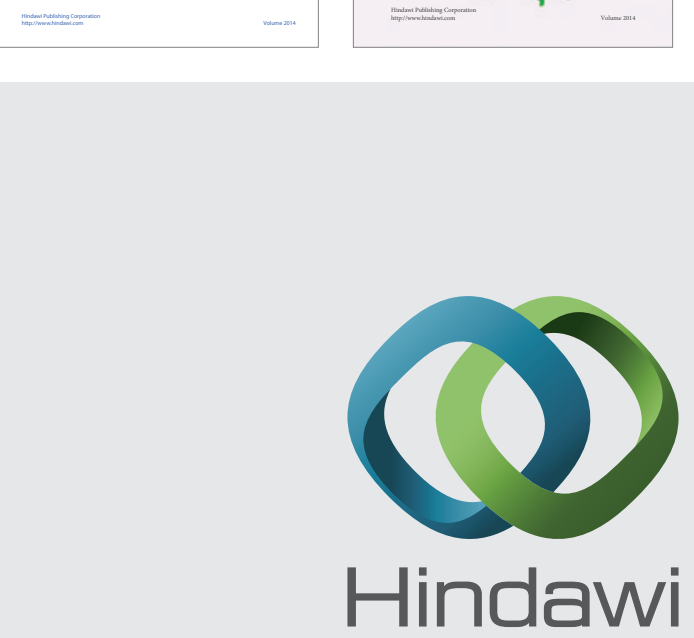

Submit your manuscripts at

http://www.hindawi.com
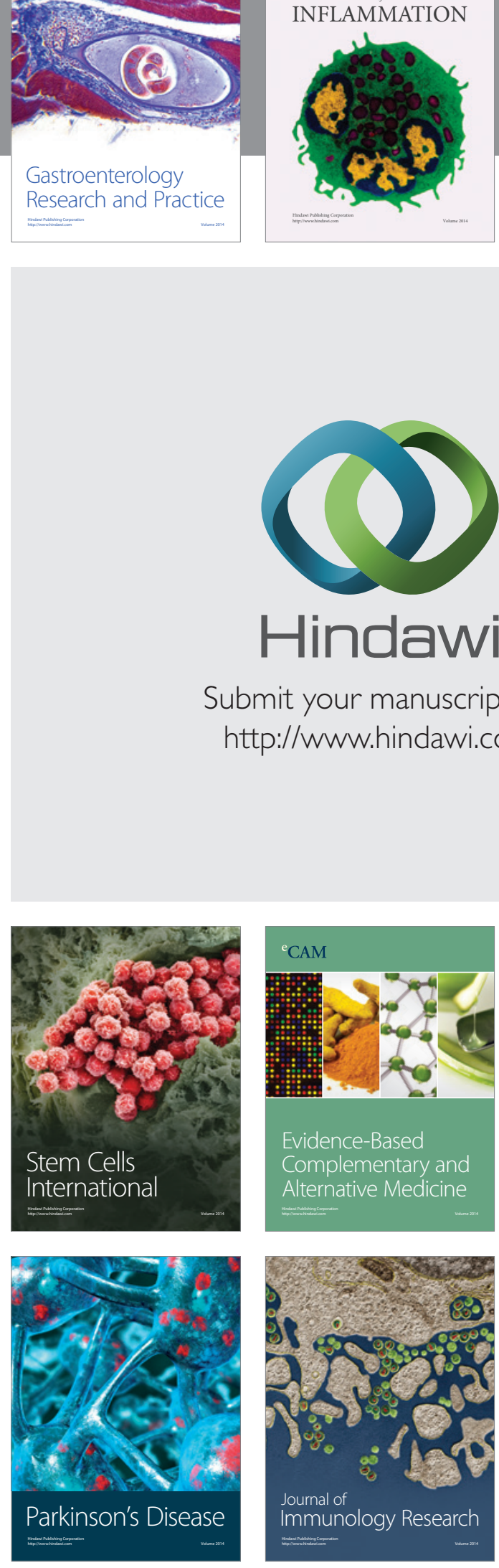

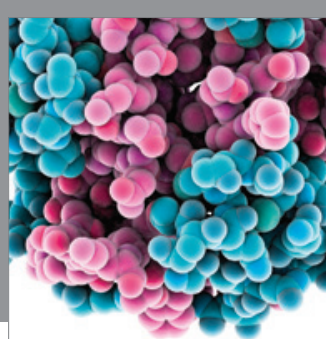

Diabetes Research
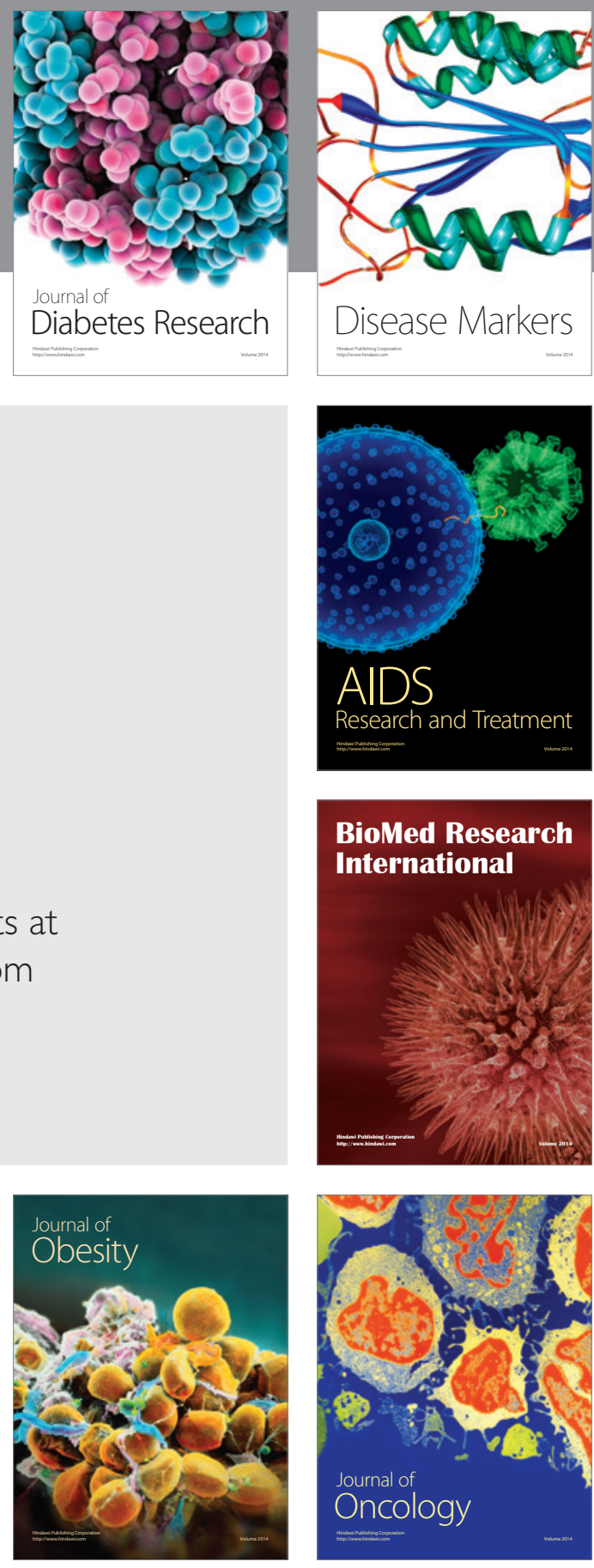

Disease Markers

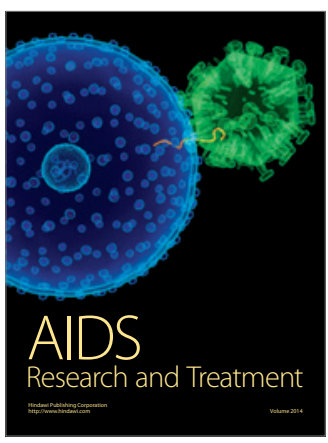

BioMed Research

International
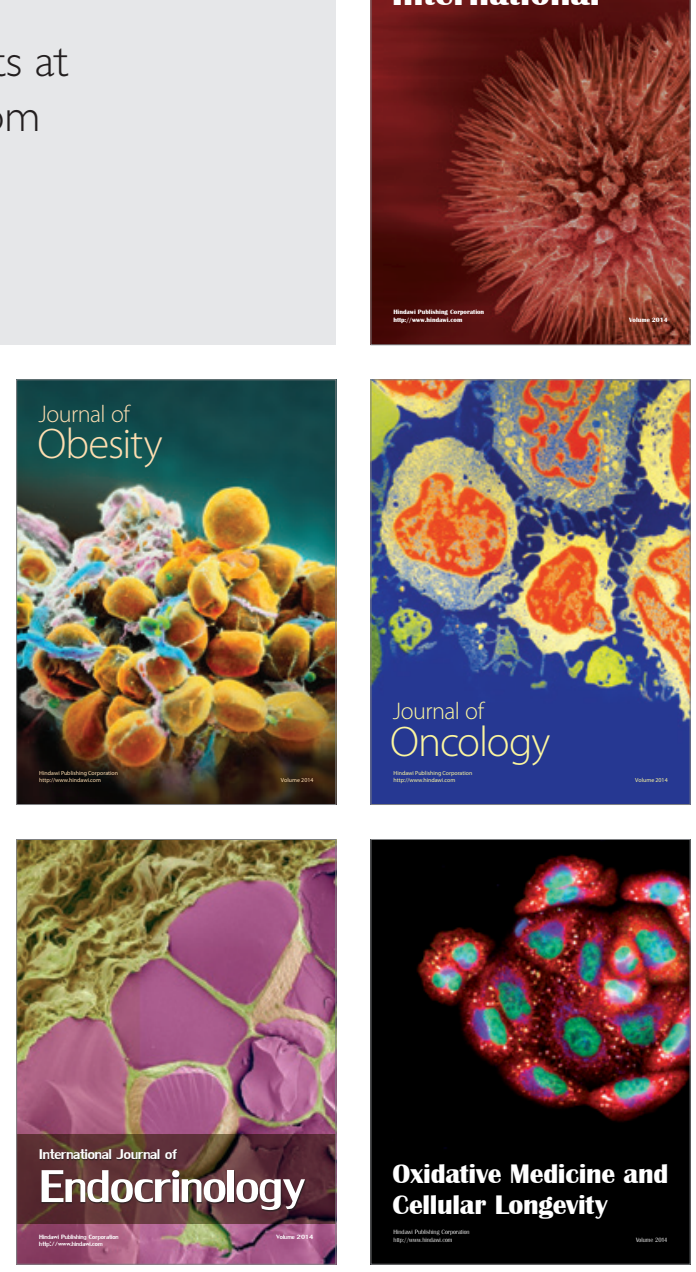